\title{
Analysis of Crack-tip Field of Particulate-Reinforced Composites Taking Account of Particle Size Effect and Debonding Damage
}

\author{
Tomoyuki Fujii' ${ }^{1, a}$, Keiichiro Tohgo, b, Yu Itoh ${ }^{2, c}$, \\ Daisuke Kato $^{1, \mathrm{~d}}$ and Yoshinobu Shimamura ${ }^{1, \mathrm{e}}$ \\ ${ }^{1}$ Department of Mechanical Engineering, Shizuoka University, 3-5-1 Johoku, Naka-ku, \\ Hamamatsu, 432-8561, Japan \\ ${ }^{2}$ Caterpillar Japan Ltd., 4-10-1 Yoga, Setagaya-ku, Tokyo 158-8530, Japan \\ ${ }^{a}$ ttfujii@ipc.shizuoka.ac.jp, ${ }^{b}$ tmktoug@ipc.shizuoka.ac.jp, ${ }^{c}$ ito_yu@cat.com \\ bdkado@mechmat.eng.shizuoka.ac.jp, etysimam@ipc.shizuoka.ac.jp
}

Keywords: Particulate-reinforced composite, Debonding damage, Size effect, Micro-mechanics, Incremental damage theory, Finite element analysis, Crack-tip field

\begin{abstract}
This paper deals with an analysis of a crack-tip field of particulate-reinforced composites which can describe the evolution of debonding damage, matrix plasticity and particle size effect on deformation and damage. Numerical analyses were carried out on a crack-tip field in elastic-plastic matrix composites reinforced with elastic particles by using a finite element method developed based on an incremental damage theory. The particle size effect on damage is described by a critical energy criterion for particle-matrix interfacial debonding. The effect of debonding damage on a crack-tip field is discussed based on numerical results. The debonding damage initiates and progresses ahead of a crack-tip. The stress distribution shifts downward in the debonding damage area. It is concluded that a crack-tip field is strongly affected by debonding damage.
\end{abstract}

\section{Introduction}

The technique to improve mechanical performance of materials by dispersing particles in a matrix has been applied to ceramic-matrix, metal-matrix and polymer-matrix composites, and these materials are called particulate-reinforced composites. A variety of damage modes such as fracture of particles and interfacial debonding between particles and matrix develop in the composites under external loading. These damage modes strongly affect mechanical performances such as stress-strain relation, tensile strength and fracture toughness [1, 2]. It is well known that mechanical performances also depend on particle size; the particle size is smaller, yield and flow stresses are more enhanced [3]. Tohgo et al. developed a constitutive model taking account of particle size effect and damage evolution and discussed the influence of debonding damage and particle size on overall stress-strain relation of composites [4]. Stress concentration area such as a crack, notch and edge in particulate-reinforced composites complicates their fracture behavior because debonding damage and matrix plasticity affect stress and strain field. To discuss the complex fracture behavior, a finite elemental analysis should be developed.

In this paper, a finite element method based on the constitutive model by Tohgo et al. [4] has been developed. Numerical analyses of a crack-tip field under three point bending are carried out for composites containing various sized particles. Influence of debonding damage on stress field ahead of a crack-tip is discussed based on the numerical results.

\section{Incremental damage theory}

The incremental damage theory of particulate-reinforced composites is briefly explained [4]. The process of debonding damage of the composites can be simulated by the following assumptions.

(1) The debonding of particles is controlled by the stress of the particles and the particle-matrix interfacial strength. (2) During debonding, the stress of the debonded particle is released and the site of the particle is regarded as a void. (3) A volume fraction of the debonded particles turns into a void 
volume fraction, and progressive damage in the composite is expressed by a decrease in an intact particle volume fraction and an increase in a void volume fraction.

Figures 1 show the states of a composite undergoing damage process before and after incremental deformation. Figure 1(a) shows the state before deformation. The volume fractions of intact particles and debonded particles are expressed by $f_{\mathrm{p}}$ and $f_{\mathrm{d}}$. If the volume fraction of the particles to be debonded in the incremental process is denoted by $d f$, the volume fractions of the intact particles and the debonded particles will change to $f_{\mathrm{p}}-d f$ and $f_{\mathrm{d}}+d f$ after incremental deformation as shown in Fig. 1(b). For this incremental deformation, the constitutive relation is described by the form decomposed into the hydrostatic and the deviatric parts as follows:

$$
\begin{aligned}
& d \varepsilon_{k k}=\frac{1}{3 \kappa_{\mathrm{t}}} d \sigma_{k k}+\frac{1}{3 \kappa_{\mathrm{d}}} \sigma_{k k}^{\mathrm{p}} d f \\
& d \varepsilon_{i j}{ }^{\prime}=\frac{1}{2 \mu_{\mathrm{t}}} d \sigma_{i j}{ }^{\prime}+\frac{1}{2 \mu_{\mathrm{d}}} \sigma^{\mathrm{p}}{ }_{i j}^{\prime} d f
\end{aligned}
$$

where $\kappa_{\mathrm{t}}, \kappa_{\mathrm{d}}, \mu_{\mathrm{t}}$ and $\mu_{\mathrm{d}}$ are the elastic moduli of the composite and are expressed as functions of the elastic moduli of the matrix and particles, $f_{\mathrm{p}}$ and $f_{\mathrm{d}}$.

Particle size effect on deformation. The theory described above is extended to consider the particle size effect by Nan-Clarke's simple method [5]. The stress-strain relation is given by Ramburg-Osgood equation as follows:

$$
\varepsilon_{\mathrm{e}}^{0}=\frac{\sigma_{\mathrm{e}}^{0}}{E_{0}}+\lambda \frac{\sigma_{0}^{0}}{E_{0}}\left(\frac{\sigma_{\mathrm{e}}^{0}}{\sigma_{0}^{0}}\right)^{1 / n}
$$

where $\sigma_{\text {e }}^{0}$ and $\varepsilon_{\text {e }}^{0}$ are the equivalent stress and strain, $E_{0}, \sigma_{0}^{0}$ and $n$ are Young's modulus, yield stress and strain-hardening exponent, respectively, and $\lambda$ is a material constant. Nan-Clarke assumed that yield stress was affected by particles in composites, and then modified as follows:

$$
\begin{aligned}
& \sigma_{0}^{0}=\left(\sigma_{0}^{0}\right)_{\text {Bulk }}+\Delta \sigma_{0}^{0} \\
& \left(\Delta \sigma_{0}^{0}\right)^{2}=\left(\Delta \sigma_{\mathrm{OR}}^{0}+\Delta \sigma_{\mathrm{KIN}}^{0}\right)^{2}+\left(\Delta \sigma_{\mathrm{ISO}}^{0}\right)^{2}+\left(\Delta \sigma_{\mathrm{CTE}}^{0}\right)^{2} \\
& =\left(\xi \mu_{0} b \sqrt{\frac{4 f_{\mathrm{p}}}{\pi d^{2}}}+\eta \mu_{0} \sqrt{\frac{f_{\mathrm{p}} \varepsilon_{\mathrm{e}}^{0 \mathrm{p}} b}{d}}\right)^{2}+\left(\zeta \mu_{0} f_{\mathrm{p}} \sqrt{\frac{\varepsilon_{\mathrm{e}}^{0 \mathrm{p}} b}{d}}\right)^{2}+\left(\gamma \mu_{0} b \sqrt{\frac{6 \Delta T \Delta \alpha f_{\mathrm{p}}}{b d\left(1-f_{\mathrm{p}}\right)}}\right)^{2}
\end{aligned}
$$

where $\mu_{0}, b, \varepsilon^{0 \mathrm{p}}$ are the shear modulus, Burgers vector and equivalent plastic strain of the matrix, and $d$ is the particle diameter. $\xi, \eta, \zeta$ and $\gamma$ are constants. $\Delta \sigma_{\text {OR }}^{0}$ is the Orowan stress, $\Delta \sigma_{\text {ISO }}^{0}$ and $\Delta \sigma_{\text {KIN }}^{0}$ are isotropic and kinematic contributions due to the effect of strain gradient plasticity, and $\Delta \sigma_{\text {CTE }}^{0}$ is a contribution of the dislocation stored in fabrication due to thermal expansion mismatch $\Delta \theta$ and temperature change $\Delta T$.

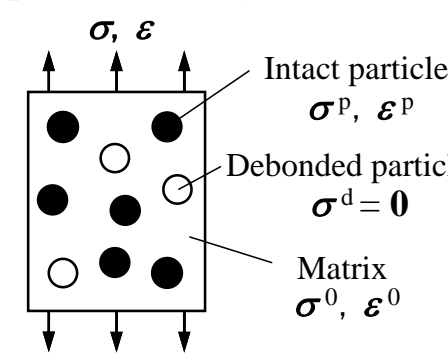

Volume fractions of intact particles: $\quad f_{\mathrm{p}}$ debonded particles: $f_{\mathrm{d}}$

(a) Before incremental deformation

Fig. 1. The states of a composite undergoing damage process before and after incremental deformation.

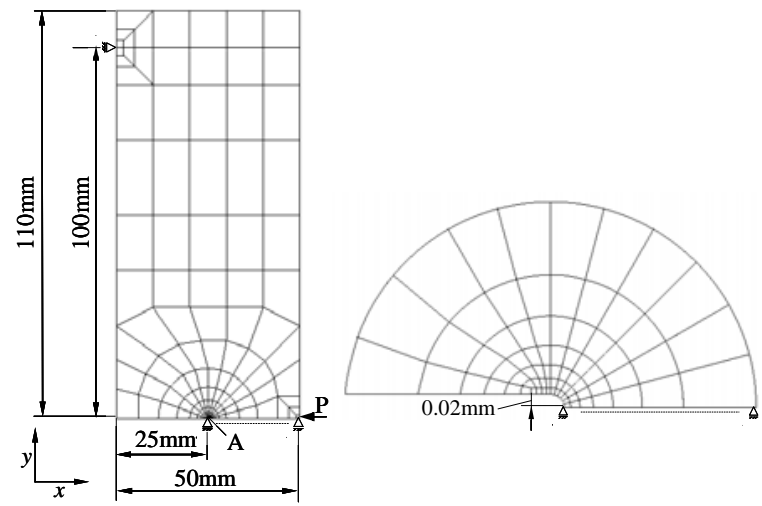

Fig. 2. Three-point-bending specimen and its finite element mesh. 
Particle size effect on debonding damage. In order to consider the particle size effect on damage, it is assumed that the debonding damage is controlled by a strain energy release rate criterion for particle-matrix interfacial debonding. From energy balance during debonding process of a particle, the critical particle stress for debonding damage is approximately given as follows:

$$
\sigma^{\mathrm{cr}}=\frac{K_{\mathrm{IC}}}{\sqrt{d}}
$$

where the fracture toughness for interfacial debonding between the particle and matrix.

Consideration of various sized particles. A number distribution of particles is assumed to follow a lognormal distribution $p(d)$,

$$
p(d)=\frac{1}{\sqrt{2 \pi} \delta d} \exp \left[-\frac{(\ln (d-\phi))^{2}}{2 \delta^{2}}\right]
$$

A composite containing various sized particles can be regarded as an assemblage of unit cells containing one particle with a constant particle volume fraction. Therefore, the influence of particles on macroscopic properties of composites does not described by the number percentage $p(d)$, but volume percentage $p_{\mathrm{v}}(d)$. The volume percentage of particle $p_{\mathrm{v}}(d)$ is given by

$$
p_{V}(d)=\frac{1}{\int d^{3} p(d) \mathrm{d} d} d^{3} p(d)
$$

Consequently, some values $A$, such as the stress, strain, particle volume fraction and so on, of a composite containing various sized particles can be obtained by the following equation,

$$
A=\int p_{V}(d) A(d) \mathrm{d} d
$$

where $A(d)$ exhibits the values for a composite with constant sized particles of $d$.

Finite element analysis based on incremental damage. Finite element method was formulated on the basis of quadrilateral 8-noded iso-parametric elements with four Gauss points. In each Gauss point, the debonding damage was calculated according to eq. (6). The yield stress was calculated on the basis of particle volume fraction using eqs. (4) and (5)

Figure 2 shows the three-point-bending specimen and its finite element mesh for a half region. In order to avoid the numerical problem for crack-tip singularity, a sharp notch with radius of $0.02 \mathrm{~mm}$ was regard as a crack. The elastic-plastic fracture mechanics parameter $J$-integral was calculated by a simple evaluation method using load-displacement relations for each stage of deformation.

Analytical condition. Numerical analysis was carried out for the composite, 15vol\%SiC/A356-T4. The material properties of matrix are $E_{0}=70 \mathrm{GPa}, v_{0}=0.33,\left(\sigma^{0}{ }_{0}\right)_{\mathrm{Bulk}}=86 \mathrm{MPa}, n=0.212, \lambda=3 / 7$, and $b=0.283 \mathrm{~nm}$. The material properties of SiC particles are $E_{\mathrm{p}}=427 \mathrm{GPa}, v_{\mathrm{p}}=0.17$ and a particle volume fraction $f_{\mathrm{p} 0}$ is $15 \%$. The thermal expansion mismatch $\Delta \theta$ and temperature change $\Delta T$ are $\theta=19.3 \times 10^{-6} /{ }^{\circ} \mathrm{C}$ and $\Delta T=520^{\circ} \mathrm{C}$. The values of $\xi, \eta, \zeta$ and $\gamma$ were $1.8,0.4,2.0$ and 1.8 , respectively. For the debonding damage, $K_{\mathrm{C}}$ was assumed to be $2.6 \mathrm{MPa} \sqrt{\mathrm{m}}$. In particle size distribution eq. (7), $\delta$ and $\phi$ were set as 0.55 and 1.864 and the average particle size was $7.5 \mu \mathrm{m}$.

\section{Analytical results and discussions}

Figure 3 shows the distributions of stress and void volume fraction ahead of a crack-tip. The solid and dotted lines are in the case of no debonding and consideration of debonding, respectively. The stress increases with progressive deformation in the case of no debonding, while the debonding initiates from a crack-tip and the stress is lower than that of no debonding in the case of consideration of debonding. The stress reduction around crack-tip is attributed to loss of load carrying capability of particle by debonding between particles and matrix. The area of debonding damage is corresponding to that of stress reduction, and the debonding damage affects the stress reduction around a crack-tip with progressive deformation. Figures 4 show the distributions of stress and void volume fraction 


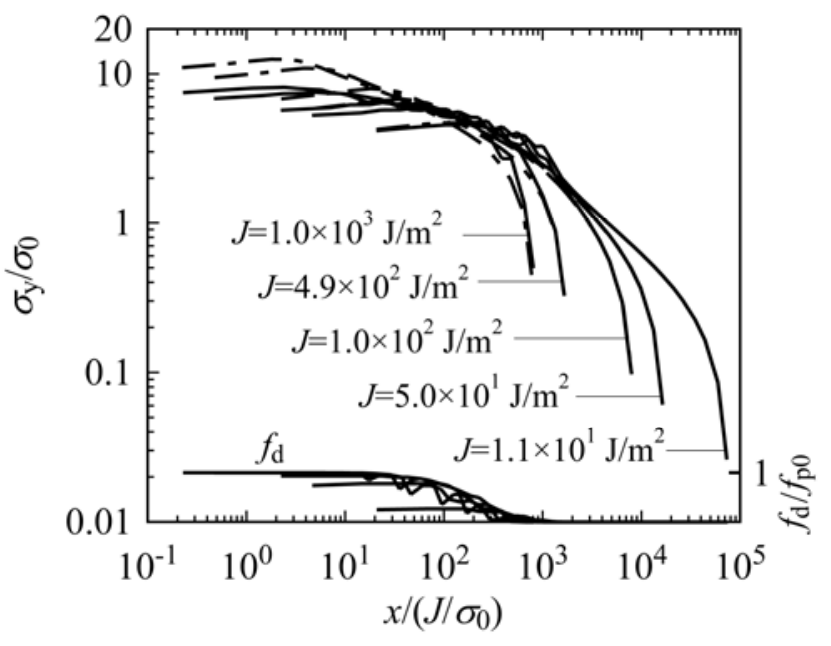

Fig. 3. Distributions of stress and void volume fraction ahead of a crack-tip.

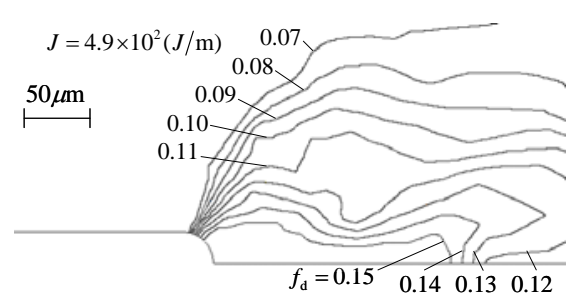

(a) Damage zone, $f_{\mathrm{d}}$

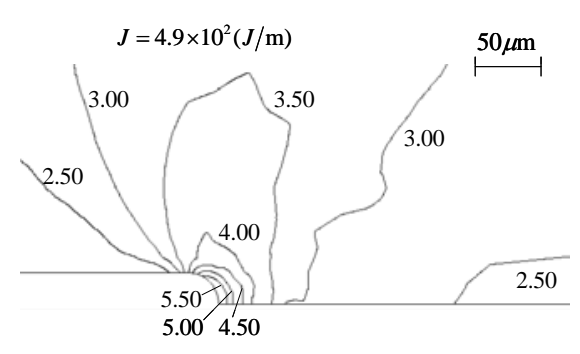

(b) Equivalent stress, $\sigma_{\mathrm{e}} / \sigma_{0}$

Fig. 4. Damage zone and equivalent stress around a crack-tip $\left(J=4.9 \times 10^{2} \mathrm{~J} / \mathrm{m}^{2}\right)$.

around a crack-tip in the case of $J=4.9 \times 10^{2} \mathrm{~J} / \mathrm{m}^{2}$. The area of void volume fraction concentrated near a crack-tip.

\section{Summary}

Numerical analyses were carried out on a crack-tip field in composites containing various sized particles taking account of the debonding damage and particle size effect. The flowing conclusions are obtained:

1. The debonding damage initiates around a crack-tip where is the stress concentration area. The stress is also reduced around a crack-tip because of loss of the load carrying capability of particle by debonding between particle and matrix.

2. The area of debonding damage and stress reduction expands around a crack-tip with progressive deformation. The debonding damage affects the stress distribution around a crack-tip.

\section{References}

[1] Kiser MT, Zok FW, Wilkinson DS, Plastic flow and fracture of a particulate metal matrix composite, Acta Materialia, 1996, Vol. 44, pp. 3465-3476.

[2] Tohgo K, Mochizuki M, Ishii H, Incremental damage theory and its application to glass-particle-reinforced Nylon 66 composites, International Journal of Mechanical Science , 1998, Vol. 40, pp.199-213.

[3] Lloyd DJ, Particle reinforced aluminum and magnesium matrix composites, International Materials Reviews, 1994, vol. 39(1), pp. 1-23.

[4] Tohgo K, Itoh Y, Shimamura Y, A constitutive model of particulate-reinforced composites taking account of particle size effects and damage evolution, Composites Part A, 2010, Vol. 41, pp. 313-321.

[5] Nan CW, Clarke DR, The influence of particle size and particle fracture on the elastic/plastic deformation of metal matrix composites, Acta Materialia, 1996, Vol. 44, pp.3801-3811. 